\title{
Correlation between Pathological Findings and the Usefulness of Clinical Guidelines for the Treatment of ANCA-Positive RPGN:
} A Retrospective Analysis

\author{
Takehisa Yamada ${ }^{1}$, Tetsuya Kashiwagi ${ }^{1}$, Akira Shimizu $^{2}$ and Shuichi Tsuruoka ${ }^{3}$ \\ ${ }^{1}$ Department of Nephrology, Nippon Medical School Chiba Hokusoh Hospital, Chiba, Japan \\ ${ }^{2}$ Department of Analytic Human Pathology, Nippon Medical School, Tokyo, Japan \\ ${ }^{3}$ Department of Nephrology, Nippon Medical School, Tokyo, Japan
}

\begin{abstract}
Background: The physical condition of patients with anti-neutrophil cytoplasmic antibody (ANCA)associated rapidly progressive glomerulonephritis (RPGN) is at times too critical for physicians to undertake a renal biopsy. In such cases, physicians need to start treatments without determining the pathological features of the disease. However, the prevalent clinical practice guidelines for ANCAassociated RPGN in Japan do not necessitate the pathological findings in a renal biopsy for determining first-line treatments. The aim of this study was to assess the correlation between the pathological findings and the clinical guideline-based treatments of ANCA-associated RPGN.

Methods: We investigated clinical and pathological features of patients who were admitted to our hospital to undergo a percutaneous renal biopsy for the diagnosis of ANCA-associated RPGN and were treated per clinical practice guidelines formulated by the Japanese Society of Nephrology. We divided patients into the following three groups according to their treatments: (a) group A, oral intake of prednisolone (PSL) only; (b) group B, methylprednisolone (mPSL) pulse therapy followed by oral intake of PSL; and (c) group C, mPSL pulse therapy followed by oral intake of PSL and the administration of immunosuppressive agents. We compared the crescent formation ratio (CFR) in glomeruli in each treatment group.

Results: The median CFR (\%; \pm standard deviation [SD]) in group A $(8.7 \% \pm 7.7 \%$ ) was significantly lower than that in groups B $(30.2 \% \pm 15.7 \%)$ and $C(71.3 \% \pm 25.5 \%)$; group A vs. group $\mathrm{B}, P<0.05$; group $\mathrm{A}$ vs. group $C, P<0.0001$. In addition, the median $C F R$ in group $B$ was significantly lower than that in group $C(P<0.01)$. The median serum creatinine levels $(\mathrm{mg} / \mathrm{dL} ; \pm \mathrm{SD})$ in both groups $\mathrm{A}(2.03 \pm 0.96 \mathrm{mg} /$ $\mathrm{dL})$ and $\mathrm{B}(1.90 \pm 0.86 \mathrm{mg} / \mathrm{dL})$ were significantly lower than that in group $\mathrm{C}(4.30 \pm 1.63 \mathrm{mg} / \mathrm{dL})$; group A vs. group $C, P<0.01$; group $B$ vs. group $C, P<0.01$. No significant difference was observed in the serum levels of C-reactive protein and ANCA in each treatment group. Although the pathological features of renal biopsy are not included in the factors in the clinical scores described in the guidelines, guidelinebased treatments reflected the severity of the crescent formation in each treatment group.

Conclusions: This study suggests that the clinical practice guidelines for ANCA-associated RPGN used in Japan facilitate physicians to determine disease management in patients presenting difficulties in undergoing a renal biopsy. (J Nippon Med Sch 2018; 85: 259-264)
\end{abstract}

Key words: ANCA-associated RPGN, treatment, pathology

\section{Introduction}

At times, the physical condition of patients with antineutrophil cytoplasmic antibody (ANCA)-associated rap- idly progressive glomerulonephritis (RPGN) is too critical for physicians to undertake a renal biopsy. Hence, the Japanese Society of Nephrology (JSN) has formulated

Correspondence to Takehisa Yamada, Department of Nephrology, Nippon Medical School Chiba Hokusoh Hospital, 1715

Kamagari, Inzai, Chiba, Japan

E-mail: t-yamada@nms.ac.jp

Journal Website (http://www2.nms.ac.jp/jnms/) 


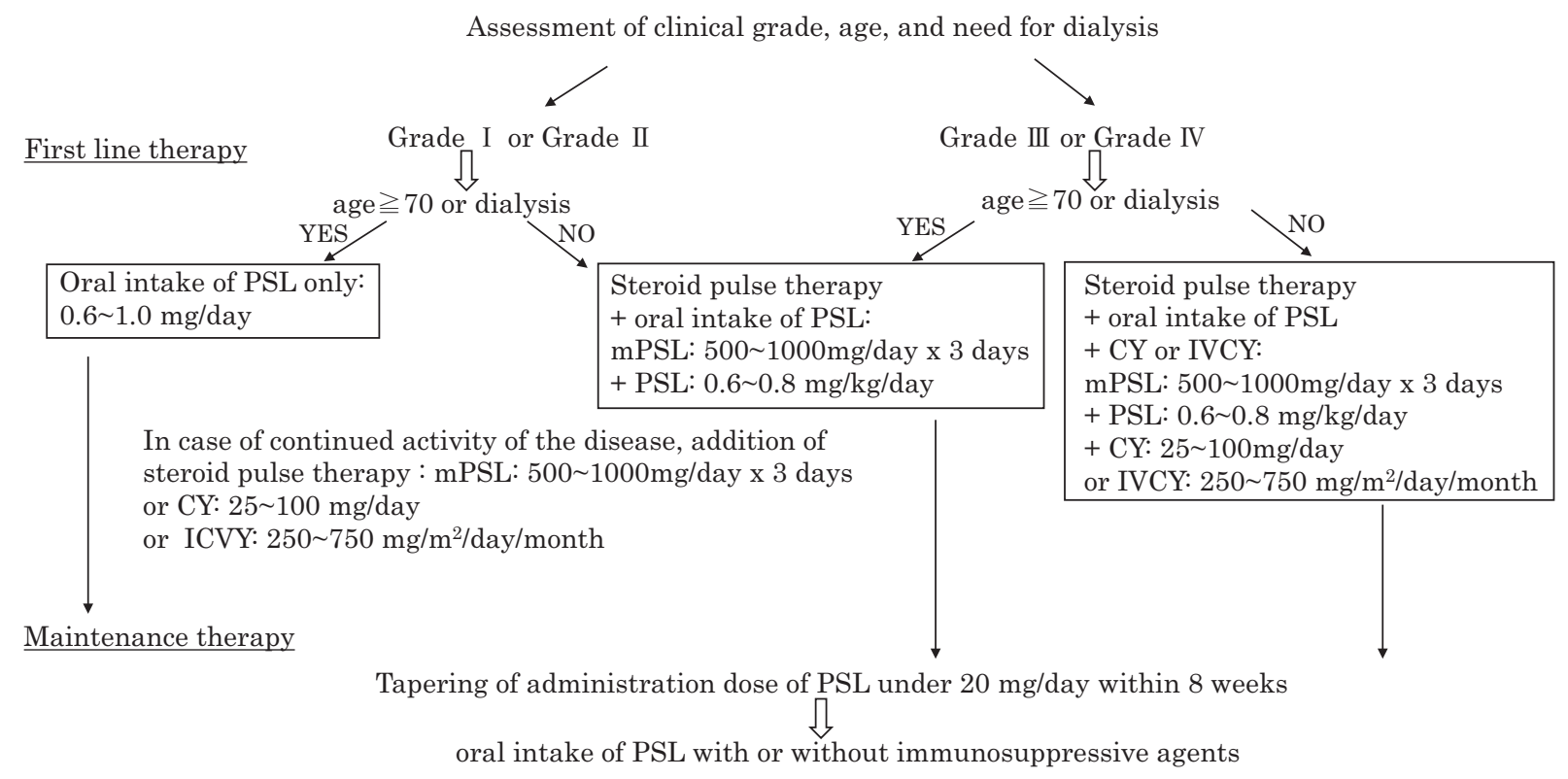

Fig. 1 The algorithm of treatment for anti-neutrophil cytoplasmic antibody (ANCA) -associated rapidly progressive glomerulonephritis (RPGN). The first-line therapy is determined by assessment of the clinical grade, age, and need for dialysis. Additional therapies are considered in case of continuation of disease activity (modified from reference 1). PSL, prednisolone; mPSL, methylprednisolone; CY, cyclophosphamide; IVCY, intravenous cyclophosphamide.

clinical practice guidelines for ANCA-associated RPGN ${ }^{1}$ to suggest first-line therapies to physicians based on a patient's clinical score, which is determined by age, presence or absence of lung diseases, and serum levels of creatinine and C-reactive protein (CRP). After that, a second-line therapy can be determined on the basis of the therapeutic response to the first-line therapy. Hence, physicians do not necessarily have to undertake a renal biopsy when they use these clinical guidelines for the treatment of patients with ANCA-associated RPGN in critical conditions. In contrast, it is imperative for physicians to assess whether the guideline-based treatments are adequate for the severity of the pathological features in patients who could have undergone a renal biopsy.

\section{Materials and Methods}

We retrospectively examined patients who were admitted to our hospital to receive a percutaneous renal biopsy for the diagnosis and treatment of ANCA-associated RPGN. We compared the clinical and pathological features of these patients in each treatment group.

\section{Patients}

We enrolled 29 patients with biopsy-proven crescentic glomerulonephritis and/or necrotizing vasculitis in our hospital. We excluded patients who were negative for ANCA or positive for anti-glomerular basement membrane antibodies. Finally, we enrolled 22 patients for analysis. All enrolled patients fulfilled the criteria for the Chapel Hill consensus conference definition of ANCAassociated vasculitis ${ }^{2}$. Furthermore, we treated all patients based on the clinical guidelines for ANCA-associated RPGN formulated by the JSN ${ }^{1}$ (Fig. 1).

\section{Informed Consent}

In this study, we followed the retrospective observational information disclosure procedure (opt-out) of the Nippon Medical School Chiba Hokusoh Hospital by obtaining informed consent from the study population. This study was approved by the Ethics Committee of the Nippon Medical School Chiba Hokusoh Hospital (no. 595).

\section{Grouping of the Patients}

We retrospectively reviewed the medical records of the enrolled patients for their age, serum levels of creatinine and CRP, and presence or absence of lung disease to determine the clinical grade described in the clinical practice guidelines ${ }^{1}$ for ANCA-associated RPGN (Table 1). We divided all patients into the following three groups based on their treatments: (a) group A, oral prednisolone (PSL) intake only; (b) group B, methylprednisolone (mPSL) pulse therapy and oral intake of PSL; and (c) group C, mPSL pulse therapy, combined with oral intake of PSL and immunosuppressive agents. Notably, all these treatments were based on the clinical guidelines for RPGN, described earlier ${ }^{1}$. 
Table 1 Categorization of the clinical grade by the total clinical scores. The clinical grade is determined by the sum of each clinical score: age, serum levels of creatinine and C-reactive protein (CRP), and presence or absence of lung disease (modified from reference 1)

\begin{tabular}{ccccc}
\hline score & $\begin{array}{c}\text { serum creatinine } \\
(\mathrm{Cr}: \mathrm{mg} / \mathrm{dL})\end{array}$ & age & lung diseases & $\begin{array}{c}\text { serum CRP } \\
(\mathrm{mg} / \mathrm{dL})\end{array}$ \\
\hline 0 & {$[\mathrm{Cr}]<3$} & $<60$ & none & $<2.6$ \\
1 & $3 \leqq[\mathrm{Cr}]<6$ & $60 \sim 69$ & & $2.6 \sim 10$ \\
2 & $6 \leqq[\mathrm{Cr}]$ & $\geqq 70$ & + & $>10$ \\
3 & dialysis & & & \\
\hline
\end{tabular}

\begin{tabular}{lc}
\hline Clinical grade & Total scores \\
\hline Grade I & $0 \sim 2$ \\
Grade II & $3 \sim 5$ \\
Grade III & $6 \sim 7$ \\
Grade IV & $8 \sim 9$ \\
\hline
\end{tabular}

Table 2 Distribution of the clinical grades in each treatment group. No significant difference was observed in the distribution of each clinical grade in each treatment group (Fisher's exact test for a $3 \times 3$ contingency table)

\begin{tabular}{lccc}
\hline & Grade I & Grade II & Grade III \\
\hline Group A (n=7) & 1 & 4 & 2 \\
Group B (n=6) & 1 & 4 & 1 \\
Group C $(\mathrm{n}=9)$ & 1 & 3 & 5 \\
\hline
\end{tabular}

Exact probability $=0.68$ (Fisher's exact test for a $3 \times 3$ contingency table)

\section{Assessment of Pathological Features in Renal Biopsy Samples}

In this study, we reviewed renal biopsies, processed using normal techniques for light microscopy, obtained from patients, and recorded the total number of glomeruli and glomeruli demonstrating crescent formation. Then, we calculated the crescent formation ratio (CFR) using the following equation:

CFR $(\%)=($ the number of crescent formation glomeruli)/(the total number of glomeruli) $\times 100$

Based on the classification system of ANCA-associated glomerulonephritis, proposed by Berden et al. ${ }^{3}$, the pathological glomerular features were categorized into four groups as follows: focal ( $\geq 50 \%$ normal glomeruli); crescentic ( $\geq 50 \%$ glomeruli with cellular crescents); mixed ( $<50 \%$ normal, $<50 \%$ crescentic, and $<50 \%$ globally sclerotic glomeruli); and sclerotic ( $\geq 50 \%$ globally sclerotic glomeruli).

\section{Survival Rate at 1 Year}

One year after the treatment, we evaluated the number of patients who were dead or alive and estimated the survival rate in each treatment group.

\section{Statistical Analysis}

While continuous variables were compared using the unpaired $t$-test, categorical variables were compared using the Fisher's exact test. We considered $P<0.05$ as statistically significant.
Table 3 Clinical characteristics of each treatment group. No significant differences were observed in the serum levels of C-reactive protein (CRP) and anti-neutrophil cytoplasmic antibody (ANCA)

\begin{tabular}{lccc}
\hline & Age (years) & $\begin{array}{c}\text { Serum CRP } \\
(\mathrm{mg} / \mathrm{dL})\end{array}$ & $\begin{array}{c}\text { ANCA } \\
(\mathrm{IU} / \mathrm{mL})\end{array}$ \\
\hline Group A $(\mathrm{n}=7)$ & $70.4 \pm 7.7$ & $10.7 \pm 5.2$ & $97.9 \pm 84.7$ \\
Group B $(\mathrm{n}=6)$ & $72.8 \pm 10.1$ & $12.7 \pm 6.4$ & $132.5 \pm 113.0$ \\
Group C $(\mathrm{n}=9)$ & $64.4 \pm 12.5$ & $11.2 \pm 5.5$ & $282.3 \pm 239.8$ \\
\hline
\end{tabular}

\section{Results}

Distribution of the Clinical Grade in Each Treatment Group

The number of patients in groups $\mathrm{A}, \mathrm{B}$, and $\mathrm{C}$ was seven, six, and nine, respectively. Each treatment group included one patient with clinical grade I. The number of patients with clinical grade II was four in both groups A and $B$, and three in group C. In addition, the number of patients with clinical grade III was two, one, and five in groups A, B, and C, respectively. Of note, there was no patient with clinical grade IV in this study. We observed no significant difference in the distribution of each clinical grade in each treatment group (Table 2).

\section{The Serum Levels of CRP and ANCA at Baseline}

The median serum CRP levels at baseline $(\mathrm{mg} / \mathrm{dL} ; \pm$ standard deviation [SD]) were $10.7 \pm 5.2 \mathrm{mg} / \mathrm{dL}$ in group A, $12.7 \pm 6.4 \mathrm{mg} / \mathrm{dL}$ in group $\mathrm{B}$, and $11.2 \pm 5.5 \mathrm{mg} / \mathrm{dL}$ in group $\mathrm{C}$. The median serum titers of ANCA at baseline $(\mathrm{IU} / \mathrm{mL} ; \pm \mathrm{SD})$ were $97.9 \pm 84.7 \mathrm{IU} / \mathrm{mL}$ in group $\mathrm{A}, 132.5 \pm$ $113.0 \mathrm{IU} / \mathrm{mL}$ in group $\mathrm{B}$, and $282.3 \pm 239.8 \mathrm{IU} / \mathrm{mL}$ in group C. No significant difference was observed in the serum levels of CRP and ANCA in each treatment group (Table 3).

Distribution of the Pathological Classification in Each Treatment Group

In this study, the number of patients with a focal type pathological classification was seven, two, and one in groups A, B, and C, respectively. The number of patients with a mixed type pathological classification was two in 
group B, and zero in groups $\mathrm{A}$ and $\mathrm{C}$. In addition, the number of patients with a crescentic type pathological classification was two and eight in groups B and C, respectively, while there was no patient with a crescentic type pathological classification in group A. Of note, there was no patient with a sclerotic type pathological classification in this study (Table 4). We observed a significant difference in the distribution of the pathological classifications in each treatment group $(P<0.001$, Fisher's exact test for a $3 \times 3$ contingency table).

CFR at Diagnosis and Serum Creatinine Levels at Baseline in Each Treatment Group

The median CFR $(\% ; \pm \mathrm{SD})$ in group A $(8.7 \% \pm 7.7 \%)$ was

Table 4 Distribution of the histological classification in each treatment group. Significant differences were noted in the distribution of the histological classification in each treatment group $(P<0.001$; Fisher's exact test for a $3 \times 3$ contingency table)

\begin{tabular}{lccc}
\hline & Focal & Mixed & Crescentic \\
\hline Group A $(n=7)$ & 7 & 0 & 0 \\
Group B $(n=6)$ & 2 & 2 & 2 \\
Group C $(n=9)$ & 1 & 0 & 8 \\
\hline
\end{tabular}

Exact probability $<0.001$ (Fisher's exact test for a $3 \times 3$ contingency table) significantly lower than that in groups B $(30.2 \% \pm 15.7 \% ; P$ $<0.05)$ and $C(71.3 \% \pm 25.5 \% ; P<0.0001)$. Furthermore, the median CFR in group $B$ was significantly lower than that in group $C(P<0.01$; Table 5; Fig. 2a).

The median serum creatinine levels $(\mathrm{mg} / \mathrm{dL} ; \pm \mathrm{SD})$ in both groups A $(2.03 \pm 0.96 \mathrm{mg} / \mathrm{dL})$ and B $(1.90 \pm 0.86 \mathrm{mg} /$ $\mathrm{dL})$ were significantly lower than that in group $C(4.30 \pm$ $1.63 \mathrm{mg} / \mathrm{dL})$; group A vs. group C, $P<0.01$; group B vs. group $C, P<0.01$. We observed no significant difference in the serum creatinine levels between groups A and B (Table 5; Fig. 2b).

\section{Survival Rate at 1 Year in Each Treatment Group}

In this study, the survival rate at 1 year was $85.7 \%$ (six

Table 5 Crescent formation ratio (CER) (\%), serum creatinine $(\mathrm{sCr})$ levels, and survival rate at 1 year in each treatment group. $\mathrm{CFR}$ and $\mathrm{sCr}$ are expressed as mean values \pm standard deviation (SD). The survival rate at 1 year in group A was $85.7 \%, 66.7 \%$ in group $\mathrm{B}$, and $77.8 \%$ in group C

\begin{tabular}{lccc}
\hline & CFR $(\%)$ & $\mathrm{sCr}(\mathrm{mg} / \mathrm{dL})$ & $\begin{array}{c}\text { Survival rate } \\
\text { at } 1 \text { year }(\%)\end{array}$ \\
\hline Group A $(\mathrm{n}=7)$ & $8.7 \pm 7.7$ & $2.03 \pm 0.96$ & 85.7 \\
Group B $(\mathrm{n}=6)$ & $30.2 \pm 15.7$ & $1.90 \pm 0.86$ & 66.7 \\
Group C $(\mathrm{n}=9)$ & $71.3 \pm 25.5$ & $4.30 \pm 1.63$ & 77.8 \\
\hline
\end{tabular}

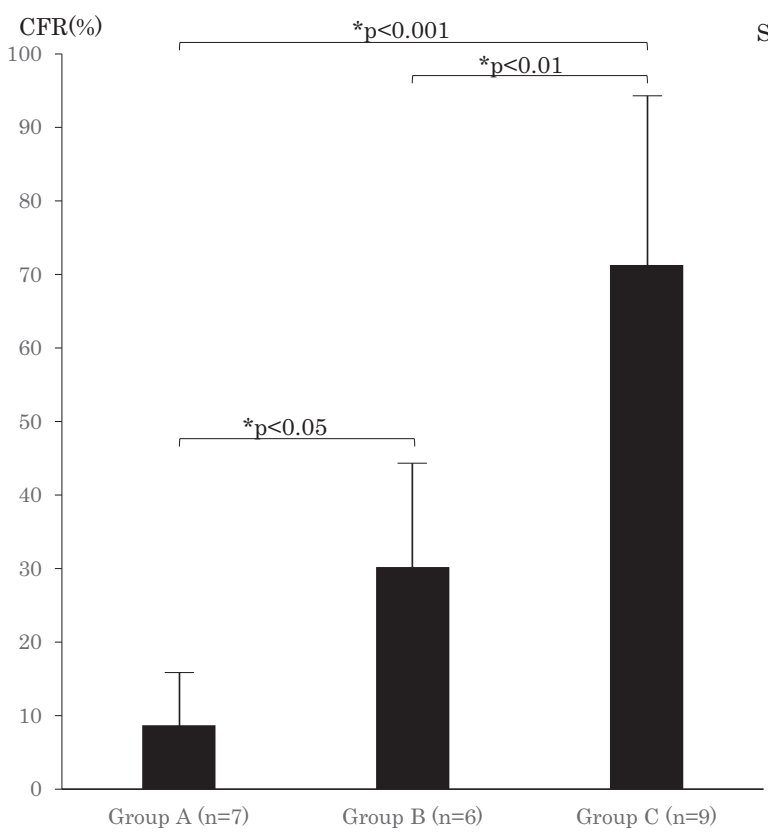

a

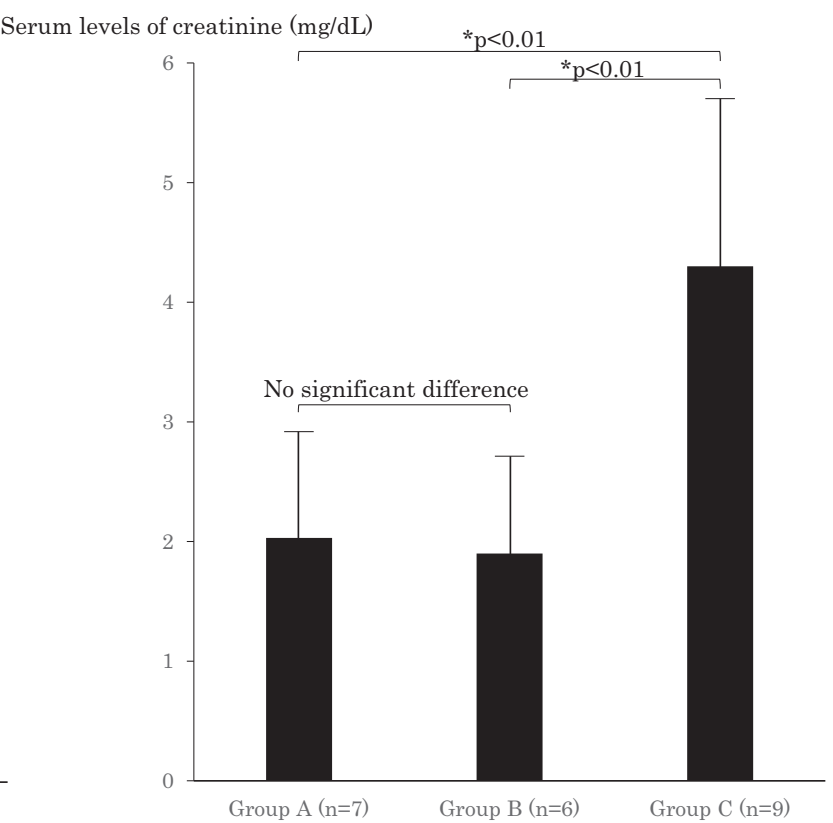

$\mathrm{b}$

Fig. 2 Crescent formation ratio (CFR) (\%) and serum creatinine levels in each treatment group. CFR in group A was significantly lower than that in group B. In addition, CFR in group A was significantly lower than that in group C, and CFR in group B was significantly lower than that in group C. The serum levels of creatinine in both groups $\mathrm{A}$ and B were significantly lower than that in group C. No significant difference was observed in the serum creatinine levels between groups $A$ and $B$. 
of seven) in group A, 66.7\% (four of six) in group B, and $77.8 \%$ (seven of nine) in group C (Table 5).

\section{Discussion}

Some studies have reported a correlation between the prognosis and the pathological features of ANCAassociated $\mathrm{RPGN}^{4-12}$, in which glomerular and tubulointerstitial lesions are predictors of prognosticate renal outcomes. Vergunst et al. ${ }^{6}$ demonstrated that these renal pathological findings in conjunction with the estimated glomerular filtration rate (eGFR) at baseline are good prognostic predictors for renal outcomes. In contrast, Berden et al. $^{3}$ proposed a new classification for ANCAassociated glomerulonephritis and highlighted the paramount importance of glomerular pathology in predicting renal outcomes. Glomerular pathological features are classified into four general categories as follows: (a) focal ( $\geq 50 \%$ normal glomeruli), (b) crescentic ( $\geq 50 \%$ glomeruli with cellular crescents), (c) mixed ( $<50 \%$ normal, < $50 \%$ crescentic, and $<50 \%$ globally sclerotic glomeruli), and (d) sclerotic ( $\geq 50 \%$ globally sclerotic glomeruli) ${ }^{3}$. After their study, some other studies revealed that the combination of this new pathological classification and other prognostic predictors, including the percentage of normal glomeruli, eGFR at baseline, myeloperoxidase-ANCA positivity, and renal tubular atrophy, is useful to predict the prognosis for renal outcomes ${ }^{8-12}$. Despite extensive research on the prognosis and pathological features of ANCA-associated $\mathrm{RPGN}^{4-12}$, limited studies have reported treatments in conjunction with the pathological features; thus, the selection criteria of treatments remain uncertain.

This study evaluated the CFR in glomeruli and compared them to treatments based on the Japanese clinical practice guidelines for ANCA-associated RPGN which recommends physicians select first-line therapies by calculating the sum of several clinical scores, including age, serum levels of creatinine and CRP, and presence or absence of lung disease ${ }^{1}$. As pathological findings obtained from renal biopsy specimens are not included in these clinical scores, these guidelines are useful for the treatment of patients with ANCA-associated RPGN whose physical conditions are too critical to undergo a renal biopsy. However, it is imperative for physicians to evaluate the adequacy of clinical guideline-based treatments from the perspective of the severity of the renal lesion in patients who are tolerant of a renal biopsy. In this study, treatments based on the guidelines reflected the severity of the crescent formation in each treatment group, although the pathological glomerular features of renal bi- opsy specimens are not involved in the factors that determine the clinical score explained in the guidelines. The treatment in group A was only an oral administration of PSL, whereas that in group B comprised an intravenous infusion of mPSL followed by an oral administration of PSL. Moreover, the treatment in group $C$ included the addition of cyclophosphamide (CY) to that in group $B$. Since the immunosuppressive factor correlated with the CFR in each treatment group, it might be possible that physicians can select the treatments that are suitable for the assumed glomerular lesion without undergoing a renal biopsy by following these guidelines. Several reports that evaluated the validation of the 2010 histological classification of ANCA-associated glomerulonephritis ${ }^{11}$ revealed that patients with a crescentic type pathological classification have a better prognosis of renal outcomes than those with a sclerotic type $\mathrm{e}^{8-12}$. In this study, the survival rate at 1 year in group $C$ was $77.8 \%$, which compared favorably with those in groups A (85.7\%) and B $(66.7 \%)$ (Table 5), whereas the ratio of patients with a crescentic type pathological classification in group $C$ was significantly higher than those in groups A and B (Table 4). These results indicate that patients with a crescentic type pathological classification could be treated with potent immunosuppressive agents and might exhibit a good prognosis, even though their CFRs were high at the time of diagnosis, if they followed the clinical practice guidelines used in Japan'. Moreover, our results revealed that patients intolerant to a renal biopsy could be treated adequately to the severity of the assumed glomerular pathological features if they followed the same guidelines. Thus, we infer that these guidelines are useful for physicians to determine the management of the disease in patients presenting difficulties in undergoing a renal biopsy.

The treatment given to two of the grade III patients and that given to one of the grade I patients was not typical when compared with the recommended therapies described in the clinical guidelines ${ }^{1}$. The clinical guidelines suggest that physicians can lower or raise the immunosuppressive factor of each recommended treatment according to the clinical condition of each patient ${ }^{1}$. Two of the grade III patients described above should not have been given immunosuppressive therapies because both of them had a severe bacterial infection of the respiratory tract. On the contrary, one of the grade I patients mentioned above was expected to have a good response to immunosuppressive agents because the patient was 34 years old and did not have any critical complications. These are the reasons why we administered only an oral 
intake of PSL to two of the grade III patients, while one of the grade I patients was treated by mPSL pulse therapy followed by oral intake of PSL and an administration of immunosuppressive agents (Table 2).

Finally, the degree of crescent formation correlated with the immunosuppressive factor in each treatment group. Based on the clinical practice guidelines, physicians could treat patients intolerant to renal biopsies in accordance with the severity of their assumed glomerular lesions because the guidelines do not mandate the pathological features in selecting the treatment. Of note, this study has the limitations of being a retrospective study and of having enrolled a small number of patients. However, to the best of our knowledge, this is the first report to assess the correlation between renal pathology and treatment in patients with ANCA-associated RPGN.

Conflict of Interest: The authors have no conflicts of interest to disclose.

\section{References}

1. Matsuo S, Kimura K, Muso E, Fujimoto S, Hasegawa M, Kaname S, Usui J, Inohara T, Kobayashi M, Itabashi M, Kitagawa K, Hirabayashi J: Clinical guideline for rapidly progressive glomerulonephritis in Japan 2014. Jpn J Nephrol 2014; 57: 139-232.

2. Jennette JC, Falk RJ, Bacon PA, Basu N, Cid MC, Ferrario F, Flores-Suarez LF, Gross WL, Guillevin L, Hagen EC, Hoffman GS, Jayne DR, Kallenberg CG, Lamprecht P, Langford CA, Luqmani RA, Mahr AD, Matteson EL, Merkel PA, Ozen S, Pusey CD, Rasmussen N, Rees AJ, Scott DG, Specks U, Stone JH, Takahashi K, Watts RA: 2012 revised international Chapel Hill consensus conference nomenclature of vasculitides. Arthritis Rheum 2013; 65: 111.

3. Berden AE, Ferrario F, Hagen EC, Jayne DR, Jennette JC, Joh K, Neumann I, Noël LH, Pusey CD, Waldherr R, Bruijn JA, Bajema IM: Histologic classification of ANCAassociated glomerulonephritis. J Am Soc Nephrol 2010; 21: $1628-1636$.

4. Bajema IM, Hagen EC, Hermans J, Noël LH, Waldherr R, Ferrario F, van der Woude FJ, Bruijn JA, for the EC/BCR project for ANCA-assay standardisation: Kidney biopsy as a predictor for renal outcome in ANCA-associated necrotizing glomerulonephritis. Kidney Int 1999; 56: 17511758 .
5. Hauer HA, Bajema IM, van Houwelingen HC, Ferrario F, Noël LH, Waldherr R, Jayne DRW, Rasmussen N, Bruijn JA, Hagen EC, for the European vasculitis study group (EUVAS): Determinants of outcome in ANCA-associated glomerulonephritis : A prospective clinicohistopathological analysis of 96 patients. Kidney Int 2002; 62: $1732-1742$.

6. Vergunst CE, van Gurp E, Hagen EC, van Houwelingen HC, Hauer HA, Noël LH, Waldherr R, Ferrario F, van der Woude FJ, Bruijn JA, Bajema IM; EC/BCR Project for ANCA-Assay Standardisation: An index for renal outcome in ANCA-associated glomerulonephritis. Am J Kid Dis 2003; 41: 532-538.

7. Day CJ, Howie AJ, Nightingale P, Shabir S, Adu D, Savage CO, Hewins P: Prediction of ESRD in pauci-immune necrotizing glomerulonephritis: quantitative histomorphometric assessment and serum creatinine. Am J Kid Dis 2010; 55: 250-258.

8. Iwakiri T, Fujimoto S, Kitagawa K, Furuichi K, Yamahana J, Matsuura Y, Yamashita A, Uezono S, Shimao Y, Hisanaga S, Tokura T, Wada T, Kitamura K, Asada Y: Validation of a newly proposed histopathological classification in Japanese patients with anti-neutrophil cytoplasmic antibody-associated glomerulonephritis. BMC Nephrol 2013; 14: 125-133.

9. Togashi M, Komatsuda A, Nara M, Omokawa A, Okuyama S, Sawada K, Wakui H: Validation of the 2010 histopathological classification of ANCA-associated glomerulonephritis in a Japanese single-center cohort. Mod Rheumatol 2014; 24: 300-303.

10. Quintana LF, Perez NS, Sousa ED, Rodas LM, Griffiths $\mathrm{MH}$, Sole M, Jayne D: ANCA serotype and histopathological classification for the prediction of renal outcome in ANCA-associated glomerulonephritis. Nephrol Dial Transplant 2014; 29: 1764-1769.

11. Ford SL, Polkinghorne KR, Longano A, Dowling J, Dayan S, Kerr PG, Holdsworth SR, Kitching AR, Summers SA: Histopathologic and clinical predictors of kidney outcomes in ANCA-associated vasculitis. Am J Kid Dis 2014; 63: $227-235$.

12. Tanna A, Guarino L, Tam FWK, Rodriquez-Cubillo B, Levy JB, Cairns TD, Griffith M, Tarzi RM, Caplin B, Salama AD, Cook T, Pusey CD: Long-term outcome of anti-neutrophil cytoplasm antibody-associated glomerulonephritis: evaluation of the international histological classification and other prognostic factors. Nephrol Dial Transplant 2015; 30: 1185-1192.

(Received, January 20, 2018)

(Accepted, March 27, 2018) 